\title{
A further investigation of visual dominance
}

\author{
FRANCIS B. COLAVITA and DANIEL WEISBERG \\ University of Pittsburgh, Pittsburgh, Pennsylvania 15260
}

\begin{abstract}
It has previously been shown that when human subjects are presented simultaneously with a light and a tone, each of which serve as a cue in a reaction time task, the subjects respond predominantly to the light. This visual dominance effect may be due to the "hard wiring" of the sensory systems involved, or the effect may be nonsensory in nature, being based on decisional or attentional factors. The present experiment suggests that visual dominance has a nonsensory basis, in that the effect is even more evident when subjects respond to stimulus offset rather than stimulus onset.
\end{abstract}

During the past several years, we have conducted a total of eight experiments dealing with the dominance of vision over audition in human subjects (Colavita, 1974; Colavita, Tomko \& Weisberg, 1976). In these experiments, subjects first matched a light and a tone for subjective magnitude. Then each stimulus was used as a cue in a choice reaction time (RT) task. Subjects pressed one response key if the tone was presented, and another key if the light was presented. On a small percentage of trials, both stimuli were delivered simultaneously. On these trials, subjects were seen to respond predominantly to the visual stimulus, being unaware in some instances that the tone had even been presented. This visual dominance effect was shown to be quite robust. It persisted when the tone was made twice as intense as the light, when subjects were instructed to respond to the tone on conflict trials, and when the tone source rather than the light source was used as a visual fixation point. The above manipulations were able to reduce, but not abolish, the subjects' tendencies to respond to the light on trials involving simultaneous light-tone presentations.

Our finding of visual dominance has recently been confirmed by Egeth and Sager (1977), utilizing a somewhat different experimental paradigm. These investigators, in addition to verifying the existence of the phenomenon, conducted further experiments in an attempt to discover the locus of the effect, i.e., whether it is a sensory phenomenon, such as some form of masking, or a nonsensory effect, such as a decisional or attentional phenomenon. Egeth and Sager concluded that the visual dominance effect is nonsensory in locus in that it is independent of stimulus intensity, but can be manipulated by instructional variables and changes in the probability of light, tone, or light-plus-tone trials. Further evidence for a nonsensory locus of the effect was the finding that in a one-key (tone key only) experimental condition, there was actually a facilitation by simultaneous light presentation of tonal RT.
Posner, Nissen, and Klein (1976) have also proposed that attentional factors serve as the basis for visual dominance. These investigators present evidence suggesting that the visual system is less well coupled to alerting mechanisms than the auditory system. Because of this relatively weak capacity of visual inputs to automatically elicit attention, we learn to direct our attention to vision. Thus, Posner et al. propose that visual dominance results from a subject's tendency to compensate for an alerting deficit present in the visual modality. In contrast to these more cognitive, decisional-attentional explanations, we have favored an explanation that is based on the "hard wiring" of the auditory and visual systems (Colavita, 1974). Specifically, we suggested that the brief stimuli used in our experiments may have been evoking a reflex orienting response, and that the orienting response to either a brief tone or a brief light involves a change in visual fixation. There is evidence (Ades, 1944) that the orienting response to either light or tone involves the neural connections of the superior colliculus with the motor centers of the ventral tegmentum, medulla, and spinal cord. Thus, we suggested that in the case of simultaneous light-tone presentations, the initial orienting tendency would favor the visual modality because of its more direct connections with the superior colliculus.

This leaves us with two proposed explanations for the visual dominance effect described above. One ascribes a nonsensory locus to the effect, implying a system that is at least under partial cognitive control (Egeth \& Sager, 1977; Posner, et al. 1976), and the other implicates the "hard wiring" of the sensory systems involved in the task (Colavita, 1974). The purpose of the present experiment was to study additional aspects of the visual dominance phenomenon in an attempt to determine which of the above explanations was most appropriate. Our procedure involved having subjects respond to stimulus offset rather than to stimulus onset, as was the case in the earlier studies. Clearly, light offset is as much a sen- 
sory event as light onset. However, Sokolov (1963) notes that the orienting response to light onset and light offset may not be of equal magnitude. The orienting response shows direct dependence on the intensity of the stimulus (p. 12). Also, there is a tendency for the electrocortical concomitants of the orienting response to diminish faster to repeated light offsets than to repeated light onsets (p. 84).

In view of the above characteristics of the orienting response, the "hard wiring" explanation of visual dominance (Colavita, 1974) and the attentional explanation (Posner et al., 1976) would seem to lead to different predictions about the dominance effect with regard to stimulus offset. The sensory explanation appears to suggest that, at best, visual dominance would remain at the same level and possibly even be diminished by having subjects respond to stimulus offset. On the other hand, the attentional explanation proposed by Posner et al. (1976) suggests that visual dominance over audition might be increased by having subjects respond to stimulus offset. This is because light offset elicits a weaker orienting response than light onset, and if an alerting deficit is responsible initially for an attentional bias toward vision, further increases in the alerting deficit (by diminishing the orienting response) should increase this attentional bias.

\section{METHOD}

The subjects for this experiment were 10 freshman psychology students from the University of Pittsburgh. The group was composed of six males and four females. All subjects were right-handed. With the exception of having subjects respond to stimulus offset rather than stimulus onset, the apparatus and procedure used was the same as those employed in our earlier work (Colavita et al., 1976). Briefly, each subject matched an auditory and a visual stimulus for subjective intensity by adjusting a $6-W$ incandescent light source until it was as bright as a $4,000-\mathrm{Hz}$, 65-dB, SPL tone was loud. Then, the offset of these matched stimuli served as cues in a $R T$ task. In a quiet room at a normal level of illumination, each subject was seated in front of a stimulus panel containing a 5-cm speaker and a $6-W$ incandescent light bulb. The subjects were instructed to look at a fixation point midway between the light and the speaker, which were $25 \mathrm{~cm}$ apart, for the duration of the experimental session. Two telegraph keys were located in front of the stimulus panel, one directly under the speaker (designated as the tone key) and one under the light (designated as the light key). The positions of the light and speaker were reversed for half of the subjects. On each trial, the light and the tone were simultaneously activated for approximately $3 \mathrm{sec}$. The subjects were instructed to press the tone key if the tone was terminated, and the light key if the light was terminated. A keypress reinstated the stimulus; then both stimuli were shut off by the experimenter, and the subject's reaction time was recorded in milliseconds. Approximately $7 \mathrm{sec}$ elapsed between trials throughout the experiment.

After four practice trials, each subject was given 20 simple RT trials when he or she was told ahead of time which stimulus would be terminated. Then, the subjects were given 20 choice RT trials when they were not told ahead of time which stimulus would be terminated. Light and tone trials were randomly presented, with the constraint that each comprise $50 \%$ of the trials.

Following each choice RT trial, the subject was asked for a verbal report as to whether or not he or she had pressed the correct key. Actually, an independent measure of correctness of key choice was available; however, the verbal report was requested in order to insure that the subjects were paying attention to their key choices.
Interspersed with the 20 choice $\mathrm{RT}$ trials were six conflict trials when both tone and light were terminated simultaneously. The subjects had not been warned ahead of time that this would be done. In the case of the conflict trials, both stimuli were reinstated when the subjects pressed either response key. The subject was also asked for a verbal report following conflict trials.

\section{RESULTS}

Mean simple RTs were $133 \mathrm{msec}$ (range $=127$ $171, \mathrm{SD}=17.3$ ) and $137 \mathrm{msec}$ (range $=123-174, \mathrm{SD}$ $=23.8$ ) for tone offset and light offset trials, respectively. These mean RT values were not significantly different from each other, but they were significantly faster $(\mathrm{p}<.001)$ than the corresponding mean RTs for tone and light onset of 269 and $271 \mathrm{msec}$ reported in an earlier study (Colavita et al., 1976), which utilized a visual fixation point midway between the speaker and the light source.

Mean RT scores for choice trials were $280 \mathrm{msec}$ (range $=210-342, \mathrm{SD}=41.4$ ) and $318 \mathrm{msec}$ (range $=264-431, \mathrm{SD}=57.7$ ) for light and tone, respectively. This difference was statistically significant (critical ratio $=2.16, p<.05$ ). There were 11 instances out of a total of 200 trials where the subjects pressed the incorrect key on a choice RT trial. One subject alone contributed five such errors, later indicating that on some trials he had been trying to "outguess" the experimenter as to which stimulus would be terminated. Each time that an error was made, the subjects were aware of it, as evidenced by their verbal reports following the trial.

A total of 60 conflict trials were presented (10 subjects, six trials each) when light and tone were terminated simultaneously. On 59 of the 60 conflict trials, the subjects pressed the light key. On no occasion was any subject aware that both stimuli had been terminated simultaneously. Even on the one conflict trial where the tone key was pressed, the subject's posttrial verbal report was that she had pressed the wrong key, that the light had been terminated, and she should have pressed the light key. Mean RT on conflict trials was $262 \mathrm{msec}$ (range = $218-323, \mathrm{SD}=36.8$ ). This value was significantly faster than the mean tonal choice RT of $318 \mathrm{msec}$ $(p<.05)$, but not significantly faster than the mean light choice RT of $280 \mathrm{msec}$.

\section{DISCUSSION}

There are a number of aspects of the present experiment that are deserving of further comment. First of all, let us look at the simple RT data. Mean RT to light or tone offset was found to be significantly faster than our previously reported simple RT values for light or tone onset. This may be because sudden onset of a stimulus produces a mild startle response with concomitant muscular contraction, whereas the termination of an ongoing stimulus may generate less of a startle reaction and thus permit a more efficient motor response. On the other hand, since collecting the data, we have become aware that the 
timer used to measure RT produced a faint auditory cue when it was activated simultaneously with light or tone offset. This auditory cue could have contaminated our simple RT data if the subjects either knowingly or unknowingly utilized it. Such a cue should have little or no effect on choice RT, in that the subject must attend to the modality of the cue stimulus in order to make the correct response. However, we are unable to make any unequivocal statements about the simple RT data.

With regard to the choice RT data, it was noted that mean RT to light offset $(280 \mathrm{msec})$ was significantly faster than mean RT to tone offset $(318 \mathrm{msec})$. This is in line with a trend seen in our earlier work (Colavita, 1974; Colavita et al., 1976) as well as in the data of Egeth and Sager (1977).

A major finding in the present experiment was the overwhelming visual dominance evidenced on conflict trials. As noted earlier, 59 light keypresses occurred out of a total of 60 conflict trials. In addition, the one tone keypress that occurred was perceived by the subjects as being incorrect. Also of interest was the fact that no subject was aware that both stimuli had been terminated simultaneously. This is actually a stronger demonstration of visual dominance than was previously reported for stimulus onset. The present data, then, appear to favor the position of Posner et al. (1976). Reducing the alerting properties of the visual stimulus by changing from onset to offset (Sokolov, 1963) seems to result in an increased attentional bias toward vision.

Our original intention had been to inform the subjects that some conflict trials would occur during a session, and that on such occasions they should press whichever key was appropriate to the signal offset they perceived first. However, a pilot subject was run without such instructions, and this subject was completely unaware that conflict trials had been administered. Thus, we continued to run subjects omitting reference to conflict trials to see how representative our pilot subject had been. We obtained data from all 10 subjects and never had a single instance of awareness of the presence of conflict trials. At the completion of testing, each subject was given complete information as to the nature of the experiment, including an explanation of the conflict trials. Each subject was asked directly whether he or she had been aware of such trials during the experiment. All subjects answered in the negative. Then, each subject was given an additional conflict trial when he or she was told it was coming. When they knew ahead of time that a conflict trial was to be presented, the subjects were able to perceive the termination of both stimuli. However, we are convinced, based on our subjects' verbal reports, that they were unaware of the occurrence of conflict trials during the actual experiment.

A possible reason for this finding of unawareness may be the fact that a key press immediately re- instated both stimuli. This may have had a distracting or disruptive (to sensory memory) effect and prevented the subjects from noting the offset of the tone.

Had the consistent finding of tonal offset unawareness not occurred, our conflict trial data would still demonstrate a strong visual dominance effect. However, the data do not support our earlier (Colavita, 1974) proposal that the effect has a sensory locus. Rather, our data provide strong support for the idea of Egeth and Sager (1977) and Posner et al. (1976) that the visual dominance effect is nonsensory in locus, being based on attentional or decisional factors. Changing the subject's task from responding to stimulus onset to responding to stimulus offset appeared to increase the visual dominance effect. No sensory "hard wiring" explanation seems adequate to deal with such a finding. Rather, an attentional, nonsensory mechanism appears to be a more likely basis for the visual dominance effect.

A final point of interest in the present study was the fact that the mean conflict RT score was $18 \mathrm{msec}$ faster than the mean choice light $\mathrm{RT}$ value, and 56 msec faster than the mean choice tone RT value. While the 18-msec difference did not achieve statistical significance, perhaps due to the relatively few conflict trials utilized, these data suggest that the offset of the tone on conflict trials may have actually had a slight facilitating effect on light key responding, even though the subjects were not aware that the tone had in fact been terminated. In this regard, our data are in accord with a typical finding in the RT to stimulus onset literature that a tonal accessory stimulus improves RT to a light (Nickerson, 1973).

Our purpose in conducting the present experiment was to obtain data which would help to clarify the nature of the visual dominance effect. The results suggest that the phenomenon has a nonsensory locus and is best explained by the attentional bias proposal of Posner et al., (1976).

\section{REFERENCES}

ADEs, H. W. Midbrain auditory mechanisms in cats. Journal of Neurophysiology, 1944, 7, 415-424.

Colavita, F. B. Human sensory dominance. Perception \& Psychophysics, 1974, 16, 409-412.

Colavita, F. B., Tomko, R., \& Weisberg, D. Visual prepotency and eye orientation. Bulletin of the Psychonomic Society, 1976, 8, 581-582.

Egeth, H. E., \& SAGer, L. C. On the locus of visual dominance. Perception \& Psychophysics, 1977, 22, 77-86.

Nickerson, R. S. Intersensory facilitation of reaction time. Psychological Review, 1973, 80, 489-509.

Posner, M. I., Nissen, M. J., \& Klein, R. M. Visual dominance: An information-processing account of its origins and significance. Psychological Review, 1976, 83, 157-171.

Sokolov, Y. N. Perception and the conditioned reflex. New York: MacMillan, 1963.

(Received for publication July 17, 1978; revision accepted January 24, 1979.) 